TRANSACTIONS OF THE

AMERICAN MATHEMATICAL SOCIETY

Volume 358, Number 11, November 2006, Pages 5059-5082

S 0002-9947(06)03908-0

Article electronically published on June 13, 2006

\title{
RATIO LIMIT THEOREM FOR PARABOLIC HORN-SHAPED DOMAINS
}

\author{
PIERRE COLLET, SERVET MARTINEZ, AND JAIME SAN MARTIN
}

\begin{abstract}
We prove that for horn-shaped domains of parabolic type, the ratio of the heat kernel at different fixed points has a limit when the time tends to infinity. We also give an explicit formula for the limit in terms of the harmonic functions.
\end{abstract}

\section{INTRODUCTION}

We consider the heat kernel in parabolic horn-shaped domains with Dirichlet boundary conditions. More precisely, we consider $D$ the domain in $\mathbf{R}^{3}$ obtained by revolving the parabolic region $\mathcal{P}=\left\{(\rho, z): 1+z^{2}<\rho\right\}$ about the $z$-axis. Thus, more explicitly we have

$$
D=\left\{\left(x^{1}, x^{2}, x^{3}\right):\left|x^{3}\right|<a\left(\sqrt{\left(x^{1}\right)^{2}+\left(x^{2}\right)^{2}}\right)\right\},
$$

where $a(t)=\sqrt{t-1}$. Although we will give a detailed proof for the case of the parabola, it is easy to verify that all our results can be extended to domains where the function $a$ satisfies the conditions of [8] together with $\infty>\lim _{s \rightarrow \infty} a(s) / s^{\gamma}>0$, $0<\gamma<1$. It is also easy to extend our results to higher dimensions.

Throughout the paper $p_{t}$ will denote the heat kernel with Dirichlet boundary conditions in $D$. The main goal of the present paper is to derive the (normalized) limit of $p_{t}$ when $t$ tends to infinity.

The case of horn-shaped domains is very different from our previous works [4, 5] since the cone $\mathcal{C}$ of nonnegative harmonic functions vanishing at the boundary is a continuum. More precisely, the Martin boundary at infinity is a circle (see [11, 8] and [13]).

The techniques are also rather different. The main point is that the associated stochastic process is at time $t$ at a distance about $t^{2 / 3}$ away from the origin, very different from the usual $t^{1 / 2}$ typical of diffusion and occurring for example in cones. As we will see below (see Theorem 1.4 and formula (5)), an important consequence is that the integral appearing in a Feynman-Kac formula is convergent, ensuring the anisotropy of the process even in the infinite time limit.

We will frequently use cylindrical coordinates defined by the axis of symmetry. The altitude will be denoted by $z$, the polar radius (for the projection in the plane $z=0$ ) will be denoted by $\rho$ and the angle (angles if we are in higher dimension) by $\vartheta$.

Received by the editors September 2, 2004 and, in revised form, November 19, 2004.

2000 Mathematics Subject Classification. Primary 60J65, 60J45, 35K05.

Key words and phrases. Bessel process, Harnack inequality, heat kernel.

(C)2006 American Mathematical Society 
We will denote by $\mathcal{R}_{\psi}$ the rotation by the angle $\psi$ around the $z$-axis. We fix once and for all a reference point $x_{0} \in D$. For example one can take this point in the plane $z=0$ at angle zero, but any other point in the domain will do equally.

We now formulate our main result. Let $v$ be the extremal of the cone $\mathcal{C}$ of harmonic functions in $D$, vanishing at the boundary, with maximal growth in the direction of $x_{0}$ (see below for the details) and satisfying $v\left(x_{0}\right)=1$. Let $v_{\psi}=v \circ \mathcal{R}_{\psi}$. Then

Theorem 1.1. For any $x$ and $y$ in $D$

$$
\lim _{t \rightarrow \infty} \frac{p_{t}(x, y)}{p_{t}\left(x_{0}, x_{0}\right)}=\frac{\int v_{\vartheta}(x) v_{\vartheta}(y) d \vartheta}{\int v_{\vartheta}\left(x_{0}\right) v_{\vartheta}\left(x_{0}\right) d \vartheta}
$$

and the limit is uniform on compact subsets of $D \times D$.

We first recall that using the same techniques as in [4] (see pages 1167-1168) one can extract from any diverging sequence of positive real numbers a diverging subsequence $\left(t_{n}\right)$ such that for any fixed $s \in \mathbf{R}$ and any $x$ and $y$ in $D$, the following limit exists:

$$
u(x, y)=\lim _{n \rightarrow \infty} \frac{p_{t_{n}+s}(x, y)}{p_{t_{n}}\left(x_{0}, x_{0}\right)} .
$$

This limit may depend on the subsequence $\left(t_{n}\right)$ (and the reference point $x_{0}$ ). We will denote it by $u(x, y)$, although $u_{\left(t_{n}\right), x_{0}}(x, y)$ would be a better notation. However, since we will only consider one subsequence at a time, there will be no possible confusion. This function is harmonic in $x$ and $y$, nonzero, and tends to zero if one of its arguments tends to the boundary of $D$ (see [4, page 1168).

The following result is the main intermediate part of the proof.

Let $q_{t}^{0}$ be the kernel defined by

$$
q_{t}^{0}(x, y)=\frac{1}{2 \pi} \int_{0}^{2 \pi} p_{t}\left(x, \mathcal{R}_{\psi} y\right) d \psi .
$$

This kernel is of course invariant by rotations about the $z$-axis in $x$ and $y$ separately.

Theorem 1.2. For any $\psi \neq 0$ (modulo $2 \pi$ ) we have

$$
\lim _{|x| \rightarrow \infty, x^{3}=0} \lim _{t \rightarrow \infty} \frac{p_{t}\left(x, \mathcal{R}_{\psi} x\right)}{q_{t}^{0}(x, x)}=0 .
$$

This result exhibits the main difference with the case of horn-shaped domains where there is only one nonnegative harmonic function zero at the boundary (hence rotation invariant) where the limit is nonzero and in fact constant.

We will use this result below in the form that the family of measures on $\psi \in \mathbf{S}^{1}$ with density $p_{t}\left(x, \mathcal{R}_{\psi} x\right) / q_{t}^{0}(x, x)$ converges weakly to the atomic measure $\delta_{0} /(2 \pi)$ at the origin.

Due to the rotation invariance of the domain, it is natural to introduce the Fourier coefficients of the heat kernel, namely for $n>0$

$$
q_{t}^{n}\left((\rho, z),\left(\rho^{\prime}, z^{\prime}\right)\right)=\frac{1}{2 \pi} \int \cos (n \psi) p_{t}\left((\rho, \psi, z),\left(\rho^{\prime}, 0, z^{\prime}\right)\right) d \psi .
$$


Theorem 1.2 will be a direct consequence of the following result.

Theorem 1.3. For any $n$ we have

$$
\lim _{\rho \rightarrow \infty} \lim _{t \rightarrow \infty} \frac{q_{t}^{n}((\rho, 0),(\rho, 0))}{q_{t}^{0}((\rho, 0),(\rho, 0))}=1 .
$$

We consider the process $Y_{t}=\left(Y^{1}(t), Y^{2}(t)\right)$, where $Y^{1}(t)$ is a Bessel process with index 2 and $Y^{2}(t)$ is an independent one-dimensional Brownian motion. Let $T$ be the first hitting time of $\mathcal{P}^{c}$. We observe that $q_{t}^{0}$ can be regarded as the transition density (with respect to the measure $\rho d \rho d z$ ) for the process $Y$ in $\mathcal{P}$ killed at the time $T$.

Theorem 1.4. We have

$$
\lim _{\rho \rightarrow \infty} \lim _{t \rightarrow \infty} \mathbf{E}_{(\rho, 0)}\left(\int_{0}^{t} \frac{d s}{Y_{s}^{1^{2}}} \mid Y_{t}=(\rho, 0), T>t\right)=0 .
$$

This result can be interpreted as saying that for the three-dimensional Brownian motion in $D$ with absorption at the boundary, the angle in cylindrical coordinates does not ergodize (see [16], Chapter V, page 186). This phenomenon was already mentioned in $[8$. We also mention here that the starting point $(\rho, 0)$ is taken for simplification, but the result holds for general $(\rho, z) \in \mathcal{P}$.

The next result gives the logarithmic behavior for the kernel $p_{t}$ and the survival probability $\mathbf{P}_{x}(T>t)$.

Theorem 1.5. For the parabolic horn-shaped domain $D$ we have

$$
\lim _{t \rightarrow \infty} t^{-1 / 3} \log \left(\mathbf{P}_{x}(T>t)\right)=2^{-2 / 3} \lim _{t \rightarrow \infty} t^{-1 / 3} \log \left(p_{t}(x, x)\right)=-C_{1 / 2},
$$

where $C_{1 / 2}$ is the constant appearing in formula (12) in [17].

The results of 17 and the second equality of the last theorem state that the asymptotic behavior for the kernel of a two-dimensional Brownian motion and the Bessel-Brownian motion kernel in the parabola have the same logarithmic decaying rate.

The rest of the paper is organized as follows. In section 2 we give a proof of Theorem 1.4. This proof is based on estimates for the large-time behavior of the process $Y$ which are given in detail in the Appendix. In section 3 we use a Fourier decomposition in the angle of the cylindrical coordinate and a FeynmanKac formula to prove Theorem 1.3 using Theorem 1.4. We then prove Theorem 1.2. In section 4 we use properties of the nonnegative harmonic functions in $D$, the Martin representation and Theorem 1.2 to finish the proof of Theorem 1.1. The Appendix is devoted to the proofs of Lemma 2.1 and Theorem 1.5 .

We observe that the results in sections 2, 3 and 4 use only the results about the Martin boundary and the estimates of Lemma 2.1. The proof therefore goes through for any domain in which these estimates can be established.

\section{Proof of Theorem 1.4}

We now start the proof of Theorem 1.4. This proof will rely on the following result for the process $Y$ in the parabola $\mathcal{P}$ defined above. We will prove below that there is up to a positive multiplicative constant one and only one harmonic function

$u^{0}$ for this process which is positive and vanishes at the boundary of the parabola 
$\mathcal{P}$. We denote by

$$
\mathcal{L}_{0}=\frac{1}{2}\left(\partial_{\rho}^{2}+\partial_{z}^{2}+\frac{1}{\rho} \partial_{\rho}\right)
$$

the generator of $Y$. Therefore $\mathcal{L}_{0} u^{0}=0$.

Lemma 2.1. For any fixed $x \in D$ the following limits exist and we have

$$
\limsup _{t \rightarrow \infty} \mathbf{E}_{(\rho, 0)}\left(\int_{0}^{t} \frac{d s}{Y_{s}^{1^{2}}} \mid Y_{t}=x, T>t\right)<\infty
$$

and

$$
\lim _{M \rightarrow \infty} \limsup _{t \rightarrow \infty} \mathbf{E}_{(\rho, 0)}\left(\int_{M}^{t-M} \frac{d s}{Y_{s}^{1^{2}}} \mid Y_{t}=x, T>t\right)=0 .
$$

This lemma is really about the position of the process $Y_{t}$ at time $t$, and roughly speaking one has $Y_{t} \approx t^{2 / 3}$ which is very different from the standard diffusion where we get $t^{1 / 2}$ whose square is not integrable.

We refer to the Appendix for a proof, and we proceed with the proof of Theorem 1.4. Let $M>0$ be fixed for the moment. We have for any $t>M$

$$
\begin{aligned}
\mathbf{E}_{(\rho, 0)} & \left(\int_{0}^{t} \frac{d s}{Y_{s}^{1^{2}}} \mid Y_{t}=(\rho, 0), T>t\right) \\
= & \mathbf{E}_{(\rho, 0)}\left(\int_{0}^{M} \frac{d s}{Y_{s}^{1^{2}}} \mid Y_{t}=(\rho, 0), T>t\right) \\
& +\mathbf{E}_{(\rho, 0)}\left(\int_{M}^{t-M} \frac{d s}{Y_{s}^{1^{2}}} \mid Y_{t}=(\rho, 0), T>t\right) \\
& +\mathbf{E}_{(\rho, 0)}\left(\int_{t-M}^{t} \frac{d s}{Y_{s}^{1^{2}}} \mid Y_{t}=(\rho, 0), T>t\right) .
\end{aligned}
$$

We will now consider the first integral, the third one being handled in the same way.

Lemma 2.2. For any fixed $x$ and $M$, we have

$$
\lim _{t \rightarrow \infty} \mathbf{E}_{(\rho, 0)}\left(\int_{0}^{M} \frac{d s}{Y_{s}^{1^{2}}} \mid Y_{t}=(\rho, 0), T>t\right)=\mathbf{E}_{(\rho, 0)}\left(\int_{0}^{M} \frac{d s}{Z_{s}^{1^{2}}}\right),
$$

where $Z$ is the limit process, namely the $u^{0}$-process of $Y$ where $u^{0}$ is the unique harmonic function for $\mathcal{L}_{0}$ (up to a constant positive factor).

Proof. We have

$$
\begin{aligned}
\mathbf{E}_{(\rho, 0)} & \left(\int_{0}^{M} \frac{d s}{Y_{s}^{1^{2}}} \mid Y_{t}=(\rho, 0), T>t\right) \\
= & \int_{0}^{M} d s \mathbf{E}_{(\rho, 0)}\left(\frac{1}{Y_{s}^{12}} \mid Y_{t}=(\rho, 0), T>t\right) .
\end{aligned}
$$

Since $Y_{s}^{1}$ is bounded below away from zero, it is enough to prove that for any fixed $s$

$$
\lim _{t \rightarrow \infty} \mathbf{E}_{(\rho, 0)}\left(\frac{1}{Y_{s}^{12}} \mid Y_{t}=(\rho, 0), T>t\right)=\mathbf{E}_{(\rho, 0)}\left(\frac{1}{Z_{s}^{12}}\right)
$$


and the result follows from Lebesgue's dominated convergence theorem. However for any $t>s$

$$
\begin{gathered}
\mathbf{E}_{(\rho, 0)}\left(\frac{1}{Y_{s}^{1^{2}}} \mid Y_{t}=(\rho, 0), T>t\right) \\
=\frac{1}{\rho q_{t}^{0}((\rho, 0),(\rho, 0))} \int_{\mathcal{P}} q_{s}^{0}((\rho, 0), y) \frac{1}{y^{1^{2}}} q_{t-s}^{0}(y,(\rho, 0)) \rho y^{1} d y .
\end{gathered}
$$

We now observe that $q^{0}$ satisfies a ratio limit theorem, namely for any $x_{1}, \ldots, x_{4}$ belonging to $\mathcal{P}$ we have

$$
\lim _{t \rightarrow \infty} \frac{q_{t}^{0}\left(x_{1}, x_{2}\right)}{q_{t}^{0}\left(x_{3}, x_{4}\right)}=\frac{u_{0}\left(x_{1}\right) u_{0}\left(x_{2}\right)}{u_{0}\left(x_{3}\right) u_{0}\left(x_{4}\right)}
$$

We refer the reader to [4 for the proof. The main ingredients are a Gaussian bound for $q_{t}^{0}$ which follows immediately from the definition and the Gaussian bound for $p_{t}$ (see also Lemma 5.1). We need Harnack's inequality and the estimate

$$
\frac{q_{t-s}^{0}((\rho, 0), y)}{q_{t}^{0}((\rho, 0),(\rho, 0))} \leq C^{\|y\|}
$$

where $C$ is a constant larger than one. This inequality can be obtained using a reflection argument for $p_{t}$, with respect to a horizontal plane, to move away from the boundary, and then apply Harnack's inequality (see Lemma 2.6 in [4 for similar computations).

We also need the uniqueness of the nonnegative harmonic function for $q^{0}$ (modulo multiplication by a constant). This last point follows immediately from the fact that any harmonic function for $q_{t}^{0}$ is a rotation invariant harmonic function for $p_{t}$. Using the Martin representation and Theorem 1 of [8] it follows that such a function is unique (up to a constant factor). One can also give a direct argument using the results of [12] on semismall perturbations.

Using this ratio limit theorem for $q^{0}$ one gets

$$
\lim _{t \rightarrow \infty} \mathbf{E}_{(\rho, 0)}\left(\frac{1}{Y_{s}^{1^{2}}} \mid Y_{t}=(\rho, 0), T>t\right)=\frac{1}{u^{0}(\rho, 0)} \int_{\mathcal{P}} q_{s}^{0}((\rho, 0), y) \frac{u^{0}(y)}{y^{1}} d y .
$$

We now investigate the limit when $M$ tends to infinity.

Lemma 2.3. For any fixed $x \in \mathcal{P}$ we have

$$
\lim _{M \rightarrow \infty} \mathbf{E}_{x}\left(\int_{0}^{M} \frac{d s}{Z_{s}^{12}}\right)<\infty
$$

and

$$
\lim _{|x| \rightarrow \infty} \mathbf{E}_{x}\left(\int_{0}^{\infty} \frac{d s}{Z_{s}^{12}}\right)=0 .
$$

Proof. The function of $M$,

$$
\mathbf{E}_{x}\left(\int_{0}^{M} \frac{d s}{Z_{s}^{12}}\right)
$$


is obviously increasing and bounded above using the first part of Lemma 2.1 and Lemma 2.2 This proves the first part. For the second part, observe that

$$
w(x)=\int_{0}^{\infty} d s \int_{\mathcal{P}} q_{s}^{0}(x, y) u^{0}(y) \frac{d y}{y^{1}}
$$

is a (finite) potential.

From the well-known Fatou-Naïm-Doob theorem (see [7] or [2] and references therein) it follows that

(even in the fine topology).

$$
\lim _{|x| \rightarrow \infty} \frac{w(x)}{u^{0}(x)}=0
$$

Theorem 1.4 now follows at once using Lemmas 2.1, 2.2 and 2.3 in formula (2).

\section{Proof of Theorems 1.2 and 1.3}

By rotation invariance around the $z$ axis, we have for any $x=(\rho, \vartheta, z), x^{\prime}=$ $\left(\rho^{\prime}, \vartheta^{\prime}, z^{\prime}\right)$ and $\psi$

$$
p_{t}\left(x, x^{\prime}\right)=p_{t}\left((\rho, \vartheta+\psi, z),\left(\rho^{\prime}, \vartheta^{\prime}+\psi, z^{\prime}\right)\right) .
$$

We now decompose in Fourier series (using also the symmetry $\vartheta \mapsto-\vartheta$ and the fact that $p_{t}$ is real), namely

$p_{t}\left((\rho, \vartheta, z),\left(\rho^{\prime}, \vartheta^{\prime}, z^{\prime}\right)\right)=q_{t}^{0}\left((\rho, z),\left(\rho^{\prime}, z^{\prime}\right)\right)+2 \sum_{n=1}^{\infty} q_{t}^{n}\left((\rho, z),\left(\rho^{\prime}, z^{\prime}\right)\right) \cos \left(n\left(\vartheta-\vartheta^{\prime}\right)\right)$.

It is easy to verify that for any $n$,

$$
\begin{gathered}
\partial_{t} q_{t}^{n}\left((\rho, z),\left(\rho^{\prime}, z^{\prime}\right)\right)=\frac{1}{2}\left(\partial_{\rho}^{2}+\frac{1}{\rho} \partial_{\rho}+\partial_{z}^{2}-\frac{n^{2}}{\rho^{2}}\right) q_{t}^{n}\left((\rho, z),\left(\rho^{\prime}, z^{\prime}\right)\right) \\
=\frac{1}{2}\left(\partial_{\rho^{\prime}}^{2}+\frac{1}{\rho^{\prime}} \partial_{\rho^{\prime}}+\partial_{z^{\prime}}^{2}-\frac{n^{2}}{\rho^{\prime 2}}\right) q_{t}^{n}\left((\rho, z),\left(\rho^{\prime}, z^{\prime}\right)\right) .
\end{gathered}
$$

Moreover, each $q_{t}^{n}$ vanishes on the boundary of the parabola (defined in the variables $\rho, z)$ and tends to the Dirac measure divided by $2 \pi$ when $t$ decreases to zero.

In other words, $2 \pi q_{t}^{n}$ is the kernel of the semi-group with infinitesimal generator

$$
\mathcal{L}_{n}=\frac{1}{2}\left(\partial_{\rho}^{2}+\frac{1}{\rho} \partial_{\rho}+\partial_{z}^{2}-\frac{n^{2}}{\rho^{2}}\right)
$$

with Dirichlet boundary conditions in the parabola and with respect to the measure $\rho d \rho d z$.

Each of these semi-groups can be represented in terms of the process $Y_{t}$ associated with the kernel $q_{t}^{0}$ using the Feynman-Kac formula, namely

$$
\frac{q_{t}^{n}\left((\rho, z),\left(\rho^{\prime}, z^{\prime}\right)\right)}{q_{t}^{0}\left((\rho, z),\left(\rho^{\prime}, z^{\prime}\right)\right)}=\mathbf{E}_{(\rho, z)}\left(e^{-\left(n^{2} / 2\right) \int_{0}^{t} \frac{d s}{Y_{s}^{12}}} \mid Y_{t}=\left(\rho^{\prime}, z^{\prime}\right), T>t\right),
$$

where $Y_{t}$ is the stochastic process associated to the kernel $q_{t}^{0}$, and $T$ the exit time from the parabola.

Observe that it follows easily from (5) that for any $n$, for any $(\rho, z) \in \mathcal{P}$ and $\left(\rho^{\prime}, z^{\prime}\right) \in \mathcal{P}$

$$
\frac{q_{t}^{n}\left((\rho, z),\left(\rho^{\prime}, z^{\prime}\right)\right)}{q_{t}^{0}\left((\rho, z),\left(\rho^{\prime}, z^{\prime}\right)\right)} \leq 1
$$


Proof of Theorem 1.3. Using Jensen's inequality in formula (5), we have

$$
\frac{q_{t}^{n}((\rho, 0),(\rho, 0))}{q_{t}^{0}((\rho, 0),(\rho, 0))} \geq e^{-\left(n^{2} / 2\right) \mathbf{E}_{(\rho, 0)}\left(\int_{0}^{t} \frac{d s}{Y_{s}^{12}} \mid Y_{t}=(\rho, 0), T>t\right)},
$$

and Theorem 1.3 follows at once from Theorem 1.4 and the upper bound ([6).

Proof of Theorem 1.2. For any fixed $x \in D$ with $x^{3}=0$, it follows easily that for any $0 \leq \psi^{\prime} \leq \psi \leq \pi$ and for any $t>0$

$$
p_{t}\left(x, \mathcal{R}_{\psi} x\right) \leq p_{t}\left(x, \mathcal{R}_{\psi^{\prime}} x\right) .
$$

This can be proved using the maximum principle or the reflection principle for Brownian Motion with respect to a vertical plane of polar angle $\phi+\left(\psi+\psi^{\prime}\right) / 2$, where $\phi$ is the polar angle of $x$.

If $\varphi$ denotes an even function on the unit circle which is twice differentiable and whose support does not contain the origin, it follows at once from Theorem 1.3 and inequality (6) that

$$
\lim _{|x| \rightarrow \infty} \lim _{t \rightarrow \infty} \int \frac{p_{t}\left(x, \mathcal{R}_{\psi} x\right)}{q_{t}^{0}(x, x)} \varphi(\psi) d \psi=0 .
$$

Now consider $\psi \in] 0, \pi]$ and let $\varphi$ be a nonnegative twice differentiable even function with support in $[\psi / 3,2 \psi / 3] \cup[-\psi / 3,-2 \psi / 3]$. We have from the monotonicity

$$
0 \leq \frac{p_{t}\left(x, \mathcal{R}_{\psi} x\right)}{q_{t}^{0}(x, x)} \int \varphi\left(\psi^{\prime}\right) d \psi^{\prime} \leq \int \frac{p_{t}\left(x, \mathcal{R}_{\psi^{\prime}} x\right)}{q_{t}^{0}(x, x)} \varphi\left(\psi^{\prime}\right) d \psi^{\prime},
$$

and Theorem 1.2 follows.

\section{Proof of Theorem 1.1}

All the extremals of the cone $\mathcal{C}$ of nonnegative harmonic functions vanishing on the boundary of $D$ can be obtained from one of them by rotation (see [11, 8] and [13]). Namely if $v$ denotes an extremal with maximal increase in the $x_{0}$ direction, we get a set of extremals $\left(v_{\vartheta}\right)$ by

$$
v_{\vartheta}=v \circ \mathcal{R}_{\vartheta},
$$

and up to a positive constant factor these are all the extremals of $\mathcal{C}$.

Lemma 4.1. For any fixed $\rho$ and $z$, the function

$$
\vartheta \rightarrow v(\rho, \vartheta, z)
$$

is nonincreasing on $[0, \pi]$ and nondecreasing on $[\pi, 2 \pi]$. We also have

$$
v(\rho, \vartheta, z)=v(\rho,-\vartheta, z)=v(\rho, 2 \pi-\vartheta, z) .
$$

Proof. By the symmetry $\vartheta \mapsto-\vartheta$, it is enough to prove the first part of the statement.

For any fixed $x \in D$, it follows easily that for any $0 \leq \psi^{\prime} \leq \psi \leq \pi$ using the maximum principle or the reflection principle for Brownian Motion with respect to a vertical plane of polar angle $\phi+\left(\psi+\psi^{\prime}\right) / 2$ where $\phi$ is the polar angle of $x$, one gets for any $s>0$

$$
p_{t}\left(s x, \mathcal{R}_{\psi} x\right) \leq p_{t}\left(s x, \mathcal{R}_{\psi^{\prime}} x\right) .
$$


Therefore the same inequality holds for the Green's function and hence also for any point of the Martin boundary corresponding to the direction of $x$ by letting $s$ tend to infinity.

Now consider one of the limiting functions $u(x, y)$ in (1). This function is harmonic in both variables $x$ and $y$ and vanishes at the boundary. Therefore we have by the Martin representation theorem [6]

$$
u(x, y)=\int v_{\vartheta}(x) v_{\varphi}(y) d \mu(\vartheta, \varphi),
$$

where $\mu$ is a positive measure on $\mathbf{S}^{1} \times \mathbf{S}^{1}$. This also follows from a result in [1] for the Martin boundary of the tensor product of two operators.

We now use the rotation symmetry around the $z$ axis to derive information about the measure $\mu$. Since for any $x, y$ and $\psi$ we have $u\left(\mathcal{R}_{\psi} x, \mathcal{R}_{\psi} y\right)=u(x, y)$, it follows from the uniqueness of the Martin representation [6] that

$$
d \mu(\vartheta, \varphi)=d \mu(\vartheta+\psi, \varphi+\psi) .
$$

It follows easily that there is a positive measure $\nu$ on $\mathbf{S}^{1}$ such that

$$
u(x, y)=\iint_{\mathbf{S}^{1} \times \mathbf{S}^{1}} v_{\psi+\eta / 2}(x) v_{\psi-\eta / 2}(y) d \psi d \nu(\eta) .
$$

We now prove a simple lemma which will be useful later on for the identification of the limit.

Lemma 4.2. Let $\left(f_{n}\right)$ be a sequence of nonnegative functions on $\mathbf{S}^{1}$, nonincreasing on $[0, \pi]$, nondecreasing on $[\pi, 2 \pi]$ and satisfying $\int f_{n}(\varphi) d \varphi=2 \pi$. Let $g$ be a nonnegative continuous function such that

$$
\lim _{n \rightarrow \infty} \int g f_{n} d \varphi=0 .
$$

Then $g(0)=0$.

Proof. If $g(0) \neq 0$, by continuity for any $\epsilon>0$ with $\epsilon<1$ there is a closed neighborhood $K_{\epsilon}$ of 0 such that for any $\varphi \in K_{\epsilon}$ we have $g(\varphi)>\epsilon g(0)$. By the monotonicity property of $f_{n}$ and its normalization we have

$$
\int_{K_{\epsilon}} f_{n}(\varphi) d \varphi \geq\left|K_{\epsilon}\right|
$$

therefore

$$
\int f_{n} g d \varphi \geq \int_{K_{\epsilon}} f_{n} g d \varphi \geq \epsilon\left|K_{\epsilon}\right| g(0),
$$

and the result follows.

Recall that $v$ is our specially chosen and normalized extremal of $\mathcal{C}$.

Lemma 4.3. For any fixed polar radius $\rho$ the function

$$
\gamma(\varphi)=\int_{0}^{2 \pi} v(\rho, \vartheta+\varphi / 2,0) v(\rho, \vartheta-\varphi / 2,0) d \vartheta
$$

is even, periodic of period $2 \pi$ and nonincreasing on $[0, \pi]$. 
Proof. We have using the relation $v(\rho, \vartheta, 0)=v(\rho, 2 \pi-\vartheta, 0)$

$$
\gamma^{\prime}(\varphi)=\int_{0}^{\pi} v(\rho, \vartheta, 0)^{\prime}[v(\rho, \vartheta-\varphi, 0)-v(\rho, \vartheta+\varphi, 0)] d \vartheta .
$$

Observe now that for $0 \leq \varphi \leq \pi$ and $0 \leq \vartheta \leq \pi$ we have

$$
v(\rho, \vartheta-\varphi, 0) \geq v(\rho, \vartheta+\varphi, 0)
$$

and since in that range of $\vartheta$ we have $v(\rho, \vartheta, 0)^{\prime} \leq 0$, we conclude that $\gamma$ is nonincreasing on $[0, \pi]$. The lemma follows using the symmetry of $v(\rho, \vartheta, 0)$ in $\vartheta=0$.

The proof of Theorem 1.1 is a direct consequence of the following proposition.

Proposition 4.4. Assume that the family of measures on $\psi \in \mathbf{S}^{1}$ with density $p_{t}\left(x, \mathcal{R}_{\psi} x\right) / q_{t}^{0}(x, x)$ converges weakly when $t \rightarrow \infty$ and then $|x| \rightarrow \infty$ to the Dirac measure at the origin. Then the measure $\nu$ is proportional to the Dirac measure at the origin.

Proof. Let $g$ be a nonnegative continuous function on $\mathbf{S}^{1}$ whose support does not contain the origin. We have for any fixed $s$ large enough

$$
\begin{gathered}
\lim _{n \rightarrow \infty} \frac{\int g(\psi) p_{t_{n}}\left(s x_{0}, s \mathcal{R}_{\psi} x_{0}\right) d \psi}{q_{t_{n}}^{0}\left(s x_{0}, s x_{0}\right)} \\
=\frac{\int v\left(s\left|x_{0}\right|, \vartheta+\varphi / 2,0\right) v\left(s\left|x_{0}\right|, \vartheta-\varphi / 2,0\right) d \vartheta d \varphi \int d \nu(\xi) g(\varphi-\xi)}{2 \pi\left(\int v\left(s\left|x_{0}\right|, \vartheta, 0\right) d \vartheta\right)^{2} \int d \nu(\xi)} .
\end{gathered}
$$

Since the function

$$
\varphi \mapsto \int d \nu(\xi) g(\varphi-\xi)
$$

is continuous, if the support of the function $g$ does not contain the origin, we get, using Lemma 4.2, Lemma 4.3 and Theorem 1.2 .

$$
\int d \nu(\xi) g(-\xi)=0
$$

and the result follows.

\section{ApPENDix}

5.1. Proof of Lemma 2.1. As mentioned earlier in the Introduction, although we will only give detailed proofs for the case of the parabola, it is easy to verify that all our results can be extended to domains of the form $\left\{\left(x^{1}, x^{2}, x^{3}\right)|| x^{3} \mid<\right.$ $\left.a\left(\sqrt{\left(x^{1}\right)^{2}+\left(x^{3}\right)^{2}}\right)\right\}$ with the function $a$ satisfying the conditions of $[8$ ] together with $\infty>\lim _{s \rightarrow \infty} a(s) / s^{\gamma}>0,0<\gamma<1$. In some of the key results below, we will make a remark on the $\gamma$ dependence of the estimates. The results can also be extended to higher dimensions.

We will denote by $r_{t}(x, y)$ the kernel in $\mathcal{P}$ given by

$$
r_{t}(x, y)=y^{1} \int_{0}^{2 \pi} p_{t}\left(\left(x^{1} \cos \theta, x^{1} \sin \theta, x^{2}\right),\left(y^{1}, 0, y^{2}\right)\right) d \theta .
$$

It is easy to verify that this is the transition kernel for the polar radius and height in cylindrical coordinates for the process defined by $p_{t}$. In particular, $r_{t}$ defines a strongly continuous semi-group. Observe that this is the transition density kernel (with respect to Lebesgue measure) for the two-dimensional process $\left(Y_{t}^{1}, Y_{t}^{2}\right)$, 
where $Y_{t}^{1}$ is a Bessel process with index $2, Y_{t}^{2}$ is a Brownian motion, and they are conditioned to $1+\left(Y_{t}^{2}\right)^{2} \leq Y_{t}^{1}$. This can also be defined using the stopping time

$$
T=\inf \left\{s>0 \mid Y_{s}^{1}=1+\left(Y_{s}^{2}\right)^{2}\right\} .
$$

The kernel $r_{t}$ is not symmetrical but satisfies

$$
\frac{r_{t}(x, y)}{y^{1}}=\frac{r_{t}(y, x)}{x^{1}}=\int_{0}^{2 \pi} p_{t}\left(\left(x^{1} \cos \theta, x^{1} \sin \theta, x^{2}\right),\left(y^{1}, 0, y^{2}\right)\right) d \theta
$$

and in particular the kernel $\left(x^{1}\right)^{1 / 2} r_{t}(x, y)\left(y^{1}\right)^{-1 / 2}$ is symmetrical. This follows at once from the definition.

The kernel $r_{t}(x, y)$ satisfies the following parabolic equation:

$$
\partial_{t} r_{t}(x, y)=\frac{1}{2}\left(\Delta_{x}+\frac{1}{x^{1}} \partial_{x^{1}}\right) r_{t}(x, y)=\frac{1}{2}\left(\Delta_{y}-\frac{1}{y^{1}} \partial_{y^{1}}+\frac{1}{\left(y^{1}\right)^{2}}\right) r_{t}(x, y),
$$

with Dirichlet boundary conditions in $\mathcal{P}$. This will allow us to apply Harnack's inequality (see for example 10]). Note that all the terms in the differential operators have the same dimension in the space variable, hence the equations are invariant by simultaneously scaling space by a factor $\lambda>0$ and time by a factor $\lambda^{2}$.

We formulate some other useful elementary facts about the kernel $r_{t}$.

Lemma 5.1. We have the Gaussian bounds

$$
\begin{gathered}
q_{t}^{0}(x, y) \leq \mathcal{O}(1) t^{-3 / 2} e^{-|x-y|^{2} /(2 t)}, \\
r_{t}(x, y) \leq \mathcal{O}(1) y^{1} t^{-3 / 2} e^{-|x-y|^{2} /(2 t)} .
\end{gathered}
$$

For fixed $x$, the function of time $t \mapsto r_{t}(x, x)$ is decreasing.

Proof. The first fact follows using the Gaussian bound for the heat kernel $p_{t}$. Indeed, using formula (7) we have

$$
\begin{gathered}
q_{t}^{0}(x, y) \leq \frac{1}{(2 \pi)^{5 / 2}(t)^{3 / 2}} e^{-\left(x^{2}-y^{2}\right)^{2} /(2 t)} \int_{0}^{2 \pi} e^{-\left[\left(x^{1} \cos \vartheta-y^{1}\right)^{2}+x^{12} \sin ^{2} \vartheta\right] /(2 t)} d \vartheta \\
=\frac{1}{(2 \pi)^{5 / 2}(t)^{3 / 2}} e^{-\left(x^{2}-y^{2}\right)^{2} /(2 t)} e^{-\left(x^{1}-y^{1}\right)^{2} /(2 t)} \int_{0}^{2 \pi} e^{-2 x^{1} y^{1}(1-\cos \vartheta) /(2 t)} d \vartheta
\end{gathered}
$$

and the result follows since $x^{1}$ and $y^{1}$ are positive. The bound on $r_{t}$ follows immediately. The second fact follows from the spectral representation of the symmetric kernel $\left(x^{1}\right)^{1 / 2} r_{t}(x, y)\left(y^{1}\right)^{-1 / 2}$ which coincides with $r_{t}(x, y)$ on the diagonal (see (4).

We will often use the sequence of boxes $\left(B_{p}\right)$ defined for $p \geq 1$ by

$$
B_{p}=\left\{\left(x^{1}, x^{2}\right) \in \mathcal{P}: p^{2} \leq x^{1} \leq(p+1)^{2}\right\} .
$$

It is also convenient to introduce the sequence of rectangles $\left(C_{p}\right)$ defined for $p \geq 1$ by

$$
C_{p}=\left\{\left(x^{1}, x^{2}\right) \in B_{p}:-p / 9 \leq x^{2} \leq p / 9\right\}
$$

and the sequence of times

$$
\tau_{p}=\sum_{0}^{p} j^{2}=\frac{p^{3}}{3}+\frac{p^{2}}{2}+\frac{p}{6} .
$$


Also for $p \geq 1$ we consider rectangles $R_{p} \subset \subset \mathcal{P}$ such that $C_{p-1} \cup C_{p} \cup C_{p+1} \subset \subset$ $R_{p}$, which for $p \geq 5$ are given by

$$
R_{p}=\left\{\left(p^{2}-3 p\right) \leq x^{1} \leq\left(p^{2}+3 p\right),-2 p / 3 \leq x^{2} \leq 2 p / 3\right\} .
$$

All the estimates below will be proved for a fixed $x$ and constants which may depend on $x$, although we will not mention this dependence explicitly. It is easy to check that these constants are uniformly bounded on compact sets.

Now, we derive an estimate on the heat kernel which is used several times later. From now on we fix a point $x \in \mathcal{P}$, which for the sake of simplicity of the following estimates we will assume $x^{2}=0$ and $x \in C_{p_{0}}$ with $p_{0} \geq 2$. A reflection argument on the second coordinate of $z=\left(z^{1}, z^{2}\right)$ shows that $r_{\tau}\left(x,\left(z^{1}, z^{2}\right)\right)$ is decreasing on $z^{2}$ (for $z^{2} \geq 0$ ). Thus we obtain for any $p>1$ and for all $\tau>0$

$$
\sup _{z \in B_{p}} r_{\tau}(x, z) \leq \sup _{z \in C_{p}} r_{\tau}(x, z) \text { and } \int_{B_{p}} r_{\tau}(x, z) d z \leq K \int_{C_{p}} r_{\tau}(x, z) d z,
$$

where $K$ is a bound for the ratio of the areas of $B_{p}$ and $C_{p}$.

For the case $p=1$, we will often use the following estimates:

$$
\sup _{z \in B_{1}} r_{\tau}(x, z) \leq 49 \sup _{z \in B_{2}} r_{\tau}(x, z) \text { and } \int_{B_{1}} r_{\tau}(x, z) \leq 49 K \int_{C_{2}} r_{\tau}(x, z) .
$$

This can be proved as follows. For fixed $x$ consider the function of $t \geq 0$ and $\left(y^{1}, y^{2}\right) \in B_{1}$ given by

$$
v\left(t, y^{1}, y^{2}\right)=\frac{r_{t}(x, y)}{\sqrt{y^{1}}} .
$$

It is easy to verify that this function satisfies

$$
\partial_{t} v=\frac{1}{2}\left[\Delta v+\frac{v}{4\left(y^{1}\right)^{2}}\right] .
$$

The function

$$
w\left(t, y^{1}, y^{2}\right)=49 v\left(t, 8-y^{1}, y^{2}\right)-v\left(t, y^{1}, y^{2}\right)
$$

satisfies in $B_{1}$ the inequality

$$
\begin{gathered}
\partial_{t} w=\frac{1}{2}\left[\Delta w+\frac{w}{4\left(y^{1}\right)^{2}}\right]+\left(\frac{49}{4\left(8-y^{1}\right)^{2}}-\frac{1}{4\left(y^{1}\right)^{2}}\right] v\left(t, 8-y^{1}, y^{2}\right) \\
\geq \frac{1}{2}\left[\Delta w+\frac{w}{4\left(y^{1}\right)^{2}}\right] .
\end{gathered}
$$

Since it is nonnegative at $t=0$ and at the boundary, we get from the maximum principle $w \geq 0$, and the result follows.

Lemma 5.2. There is a constant $C>1$ such that for any $p \geq 2, q \geq 2$ and $\tau>0$ we have

$$
\sup _{z \in C_{p}} r_{\tau}(x, z) \leq C^{1+|p-q|+\max \left\{0, p^{2}-\tau\right\} / \tau} \inf _{y \in C_{q}} r_{\max \left\{\tau, p^{2}\right\}+\left|\tau_{p}-\tau_{q}\right|+\mathbf{1}_{p=q} p^{2}}(x, y) .
$$

Moreover there are two constants $\alpha>0$ and $\beta>0$ such that for any $t>0$

$$
r_{t}(x, x) \geq \alpha e^{-\beta t^{1 / 3}} .
$$


Remarks. It is easy to verify that the constants of the previous lemma can be chosen uniformly on $x$ in compact subsets of $\mathcal{P}$. Moreover we can always assume that $\alpha=1$. For the general $\gamma \in(0,1)$ one gets a lower bound $r_{t}(x, x) \geq$ $\mathcal{O}(1) \exp \left(-\mathcal{O}(1) t^{(1-\gamma) /(1+\gamma)}\right)$.

For the case of the Dirichlet heat kernel inside the parabola, a more precise result than the second part of Lemma 5.2 has been proved in [17]. For the asymptotic tail of the survival probability, see [9] and [3].

Proof. We first consider the case $\tau \geq p^{2}$. We can apply Harnack's inequality in $R_{p}$ to conclude that there is a constant $C$ independent of $p$ such that

$$
\sup _{z \in C_{p}} r_{\tau}(x, z) \leq C \inf _{z \in R_{p}} r_{\tau+p^{2}}(x, z) .
$$

Hence the inequality holds for this case when $q=p$. If $q>p$, we use this inequality to go from $C_{p}$ to $C_{p+1}$, and if $q<p$ we go from $C_{p}$ to $C_{p-1}$. After a finite number of steps, we reach $C_{q}$ and the result follows in that case.

We now consider the case $\tau<p^{2}$. We first apply many times Harnack's inequality in a fixed rectangle of size $\sqrt{\tau} / 4$ centered in $z$. We obtain

$$
r_{\tau}(x, z) \leq C^{\left(p^{2}-\tau\right) / \tau} r_{p^{2}}(x, z) .
$$

We can now proceed as before, and the first result follows.

To prove the second inequality, using the monotonicity on time of $r .(x, x)$, we can assume $t=2 \tau_{n}+(n+1)^{2}$ for some integer $n \geq 1$.

Using the Markov property iteratively, we get

$$
\begin{gathered}
r_{t}(x, x)= \\
\int r_{1}\left(x, y_{1}\right) \cdots r_{n^{2}}\left(y_{n-1}, y_{n}\right) r_{(n+1)^{2}}\left(y_{n}, y_{n+1}\right) r_{n^{2}}\left(y_{n+1}, y_{n+2}\right) \cdots r_{1}\left(y_{2 n}, x\right) \prod_{j=1}^{2 n} d y_{j} .
\end{gathered}
$$

To get a lower bound, we restrict the integration over $y_{j}$ to $C_{j}$ if $1 \leq j \leq n$ and to $C_{2 n-j+1}$ for $n+1 \leq j \leq 2 n$. We get (from the positivity of the heat kernel)

$$
r_{t}(x, x)>\int_{\mathcal{C}(n)} \prod_{j=1}^{n+1} r_{j^{2}}\left(y_{j-1}, y_{j}\right) \prod_{j=n+1}^{2 n} r_{(2 n+1-j)^{2}}\left(y_{j}, y_{j+1}\right) d y_{1} \cdots d y_{2 n},
$$

where $\mathcal{C}(n):=C_{1} \times C_{2} \cdots C_{n} \times C_{n+1} \times C_{n} \cdots \times C_{2} \times C_{1}$ and $y_{0}=y_{2 n+1}=x$.

We now observe that for $1 \leq p \leq n$ we have for $y \in C_{p}$ and $z \in C_{p-1} \cup C_{p} \cup C_{p+1}$

$$
r_{t}(y, z) \geq r_{t}^{R_{p}}(y, z)
$$

where $r_{t}^{R_{p}}(y, z)$ denotes the kernel of the semi-group with the same generator as $r_{t}$ but with Dirichlet boundary conditions in $R_{p}$. We also observe that by scaling there is a constant $\sigma>0$ independent of $p$ such that

$$
\inf \left\{\int_{C_{p}} r_{p^{2}}^{R_{p}}(z, y) d y: z \in C_{p-1} \cup C_{p} \cup C_{p+1}\right\} \geq \sigma .
$$

We eventually choose $\sigma$ smaller such that also $\min \left\{r_{1}(z, x): z \in C_{1}\right\} \geq \sigma$, from which the result follows.

Remark. This argument is reminiscent of the so-called super-stability arguments of D. Ruelle [15] with the difference that the present setting is not extensive. 
Lemma 5.3. There are constants $a>0, b>0$ and $c>0$ such that for any $t>0$ and for any $0 \leq s \leq t / 2$ we have

$$
\mathbf{P}_{x}\left(Y_{s}^{1}>a s^{2 / 3} \mid T>t, X_{t}=x\right) \leq c e^{-b s^{1 / 3}} .
$$

Proof. Assume that $x \in C_{r_{0}}$. Let $r=\left[a^{1 / 2} s^{1 / 3}\right]-1$ for some $a>0$ large enough but independent of $s$ and $t$, such that if $p \geq r$ we have $\tau_{p}-\tau_{r_{0}}>s$. Also consider $s_{0}>1$ large enough such that $r \leq \sqrt{t-s}$ for all $s \geq s_{0}$, and $t>2 s$. By choosing $c$ large enough, it suffices to prove the result for $s \geq s_{0}$ (and $t>2 s_{0}$ ). We first estimate for an integer $p$ the conditional probability

$$
\mathbf{P}_{x}\left(Y_{s} \in B_{p} \mid T>t, X_{t}=x\right) .
$$

Using the Markov property, we have

$$
\mathbf{P}_{x}\left(Y_{s} \in B_{p} \mid T>t, X_{t}=x\right)=\frac{1}{r_{t}(x, x)} \int_{z \in B_{p}} r_{s}(x, z) r_{t-s}(z, x) d z .
$$

Our goal now is to bound the numerator in terms of the denominator. Using Lemma 5.1 (for $s>1$ ) and formula (8), we have for $p>1$

$$
\begin{gathered}
\int_{B_{p}} r_{s}(x, z) r_{t-s}(z, x) d z \leq \mathcal{O}(1) p^{4} e^{-p^{4} /(3 s)} \sup _{z \in B_{p}} r_{t-s}(z, x) \\
\leq \mathcal{O}(1) p^{4} e^{-p^{4} /(3 s)} \sup _{z \in C_{p}} r_{t-s}(z, x) .
\end{gathered}
$$

In the case $p=1$ we get similar estimates using (9).

We can now use Lemma 5.2 and the monotonicity of $r .(x, x)$ to conclude that

$$
\begin{gathered}
\sup _{z \in C_{p}} r_{t-s}(z, x) \leq \mathcal{O}(1) \sup _{z \in C_{p}} r_{t-s}(x, z) \\
\leq \mathcal{O}(1) C^{\left|p-r_{0}\right|+\max \left\{0, p^{2}-t+s\right\} /(t-s)} r_{\max \left\{t-s, p^{2}\right\}+\left|\tau_{p}-\tau_{r_{0}}\right|}(x, x) .
\end{gathered}
$$

If $p^{2}<t-s$ and since $\tau_{p}-\tau_{r_{0}}>s$ we get, again using the monotonicity of $r$. $(x, x)$,

$$
\int_{B_{p}} r_{s}(x, z) r_{t-s}(z, x) d z \leq \mathcal{O}(1) p^{4} e^{-p^{4} /(3 s)} C^{\left|p-r_{0}\right|} r_{t}(x, x) .
$$

If $p^{2}>t-s$ we get

$$
\int_{B_{p}} r_{s}(x, z) r_{t-s}(z, x) d z \leq \mathcal{O}(1) p^{4} e^{-p^{4} / 3 s} C^{\left|p-p_{0}\right|+\left(p^{2}-t+s\right) /(t-s)} r_{t}(x, x) .
$$

We now use the decomposition

$$
\begin{gathered}
\int_{z^{1}>r^{2}} r_{s}(x, z) r_{t-s}(z, x) d z=\sum_{p=r}^{\infty} \int_{B_{p}} r_{s}(x, z) r_{t-s}(z, x) d z \\
=\sum_{p=r}^{[\sqrt{t-s}} \int_{B_{p}} r_{s}(x, z) r_{t-s}(z, x) d z+\sum_{p=[\sqrt{t-s}]+1}^{\infty} \int_{B_{p}} r_{s}(x, z) r_{t-s}(z, x) d z .
\end{gathered}
$$


Using the two above bounds we have

$$
\begin{gathered}
\sum_{p=r}^{[\sqrt{t-s}]} \int_{B_{p}} r_{s}(x, z) r_{t-s}(z, x) d z \\
\leq \mathcal{O}(1) \sum_{p=r}^{[\sqrt{t-s}]} p^{4} e^{-p^{4} /(3 s)} C^{\left|p-r_{0}\right|} r_{t}(x, x) \leq \mathcal{O}(1) e^{-b s^{1 / 3}} r_{t}(x, x) .
\end{gathered}
$$

For the second sum, we observe that

$$
\begin{gathered}
\sum_{p=[\sqrt{t-s}]+1}^{\infty} \int_{B_{p}} r_{s}(x, z) r_{t-s}(z, x) d z \\
\leq \mathcal{O}(1) \sum_{p=[\sqrt{t-s}]+1}^{\infty} p^{4} e^{-p^{4} / 3 s} C^{\left|p-r_{0}\right|+\left(p^{2}-t+s\right) /(t-s)} r_{t}(x, x) .
\end{gathered}
$$

Since $t-s>s$, we have

$$
e^{-p^{4} / 6 s} C^{p^{2} /(t-s)} \leq e^{-p^{4} / 6 s} C^{p^{2} / s} \leq \mathcal{O}(1),
$$

and we can estimate the sum as before.

Lemma 5.4. For any $x \in \mathcal{P}$, there are constants $E>0$ and $D>0$ such that for any $t>2$ and for any $0 \leq s \leq t / 2$ we have

$$
r_{t-s}(x, x) \leq D e^{E s t^{-2 / 3}} r_{t}(x, x) .
$$

Remarks. Consider the function $f$ defined for $t>0$ by

$$
f(t)=e^{-a t^{1 / 3}},
$$

where $a$ is a positive constant. If $s \ll t$ we have

$$
f(t-s)=e^{-a t^{1 / 3}(1-s / t)^{1 / 3}} \approx f(t) e^{a s t^{-2 / 3} / 3},
$$

and this is in essence what the lemma says.

Note also that a similar estimate follows from Harnack's inequality, but with much worse constants.

For the general $\gamma \in(0,1)$ one gets an upper bound by

$$
r_{t-s}(x, x) \leq \mathcal{O}(1) e^{\mathcal{O}(1) s t^{-2 \gamma /(1+\gamma)}} r_{t}(x, x) .
$$

Proof. The function $t \mapsto r_{t}(x, x)$ is log-convex (see [4]), therefore for any $0 \leq s \leq$ $t / 2$ we have (since $t>2$ )

$$
\begin{gathered}
r_{t-s}(x, x) \leq r_{1}(x, x)^{s /(t-1)} r_{t}(x, x)^{(t-s-1) /(t-1)} \\
=r_{1}(x, x)^{s /(t-1)} r_{t}(x, x)^{-s /(t-1)} r_{t}(x, x),
\end{gathered}
$$

and the result follows immediately using $s /(t-1)<2$ and the second part of Lemma 5.2 . 
Lemma 5.5. There are constants $a>1$ and $A>1$ such that for any $t / 2>s^{\prime}>$ $s+1>2$, for any $p^{2}<a s^{2 / 3}$, and for any $q^{2}<a s^{2 / 3}$ with $|p-q| \geq 2$, we have

$$
\begin{gathered}
\quad \mathbf{P}_{x}\left(Y_{s} \in B_{p}, Y_{s^{\prime}} \in B_{q} \mid T>t, X_{t}=x\right) \\
\leq A q^{4} e^{-\left(p^{2}-q^{2}\right)^{2} /\left(18\left(s^{\prime}-s\right)\right)} e^{A|p-q|} e^{A\left(s^{\prime}-s\right) t^{-2 / 3}} .
\end{gathered}
$$

Proof. Observe first that if $p^{2} \leq y^{1} \leq(p+1)^{2}$ and $q^{2} \leq z^{1} \leq(q+1)^{2}$, then for $|q-p| \geq 2$ we have

$$
\left(y^{1}-z^{1}\right)^{2} \geq \frac{\left(q^{2}-p^{2}\right)^{2}}{4} .
$$

For $y \in B_{p}$ and $z \in B_{q}$ we have from Lemma 5.1

$$
\begin{aligned}
& \int_{B_{q}} r_{s^{\prime}-s}(y, z) d z \leq \mathcal{O}(1) q^{2} \int_{B_{q}} e^{-\left(\left(y^{1}-z^{1}\right)^{2}+\left(y^{2}-z^{2}\right)^{2}\right) /\left(2\left(s^{\prime}-s\right)\right)} d z \\
\leq & \mathcal{O}(1) q^{2} e^{-\left(q^{2}-p^{2}\right)^{2} /\left(9\left(s^{\prime}-s\right)\right)} \int_{B_{q}} d z \leq \mathcal{O}(1) q^{4} e^{-\left(q^{2}-p^{2}\right)^{2} /\left(18\left(s^{\prime}-s\right)\right) .}
\end{aligned}
$$

Using the Markov property and this Gaussian bound we get the following upper bound:

$$
\begin{gathered}
\mathbf{P}_{x}\left(Y_{s} \in B_{p}, Y_{s^{\prime}} \in B_{q}, T>t, Y_{t}=x\right) \\
\leq \mathcal{O}(1) q^{4} e^{-\left(p^{2}-q^{2}\right)^{2} /\left(18\left(s^{\prime}-s\right)\right)} \int_{B_{p}} r_{s}(x, y) d y \sup _{z \in B_{q}} r_{t-s^{\prime}}(z, x) \\
\leq \mathcal{O}(1) q^{4} e^{-\left(p^{2}-q^{2}\right)^{2} /\left(18\left(s^{\prime}-s\right)\right)} \int_{B_{p}} r_{s}(x, y) d y \sup _{z \in C_{q}} r_{t-s^{\prime}}(z, x) \\
\leq \mathcal{O}(1) q^{4} e^{-\left(p^{2}-q^{2}\right)^{2} /\left(18\left(s^{\prime}-s\right)\right)} \int_{C_{p}} r_{s}(x, y) d y \sup _{z \in C_{q}} r_{t-s^{\prime}}(z, x) .
\end{gathered}
$$

We now move $z$ to $y$ using Harnack's inequality if $p>1$ and $q>1$. We get from Lemma 5.2 (note that $\left.q^{2}<\mathcal{O}(1)\left(t-s^{\prime}\right)\right)$

$$
\sup _{z \in C_{q}} r_{t-s^{\prime}}(z, x) \leq C^{1+|p-q|} \inf _{z \in C_{p}} r_{\max \left(t-s^{\prime}, q^{2}\right)+\left|\tau_{q}-\tau_{p}\right|}(z, x) .
$$

We now observe that

$$
\begin{gathered}
\int_{C_{p}} r_{s}(x, y) d y \inf _{z \in C_{p}} r_{\max \left(t-s^{\prime}, q^{2}\right)+\left|\tau_{q}-\tau_{p}\right|}(z, x) \\
\leq \int_{C_{p}} r_{s}(x, y) r_{\max \left(t-s^{\prime}, q^{2}\right)+\left|\tau_{q}-\tau_{p}\right|}(y, x) d y \\
\leq r_{\max \left(t-s^{\prime}, q^{2}\right)+\left|\tau_{q}-\tau_{p}\right|+s}(x, x) \leq r_{t+\left|\tau_{q}-\tau_{p}\right|+s-s^{\prime}}(x, x)
\end{gathered}
$$

by monotonicity on time of $r$. $(x, x)$. If $\left|\tau_{q}-\tau_{p}\right| \geq s^{\prime}-s$ this is smaller than $r_{t}(x, x)$ by monotonicity on time.

Finally, using Lemma 5.4, the result follows since in the cases $p=1$ and/or $q=1$ we can first use the estimates (9) to come back to the previous cases. 
Lemma 5.6. There are constants $A^{\prime}>0, C^{\prime}>0$ and $h_{0}>0$ such that for any $0 \leq s \leq t / 2$ we have for any $h>h_{0}$

$$
\begin{gathered}
\mathbf{P}_{x}\left(\sup _{s-s^{2 / 3} \leq \tau \leq s}\left|Y_{\tau}^{1}-Y_{s}^{1}\right|>h s^{4 / 9} \mid T>t, X_{t}=x\right) \\
\leq C^{\prime} s^{2} e^{-A^{\prime} h^{2} s^{2 / 9}}+C^{\prime} e^{-A^{\prime} s^{1 / 3}} .
\end{gathered}
$$

Proof. As in the previous proofs, we may assume $s \geq s_{0}$, with $s_{0}$ large enough. We also take $s^{\prime}=s+s^{2 / 3}$. We can write

$$
\begin{gathered}
\mathbf{P}_{x}\left(\sup _{s-s^{2 / 3} \leq \tau \leq s}\left|Y_{\tau}^{1}-Y_{s}^{1}\right|>h s^{4 / 9} \mid T>t, X_{t}=x\right) \\
=\sum_{p, q} \mathbf{P}_{x}\left(\sup _{s-s^{2 / 3} \leq \tau \leq s}\left|Y_{\tau}^{1}-Y_{s}^{1}\right|>h s^{4 / 9}, Y_{s-s^{2 / 3}} \in B_{p}, Y_{s} \in B_{q} \mid T>t, X_{t}=x\right) .
\end{gathered}
$$

Using Lemma 5.3, it is enough to consider in the above infinite sum only the terms with $p^{2}<a s^{2 / 3}$ and $q^{2}<a s^{2 / 3}$. This gives an error corresponding to the second term in the estimate.

If $\left|p^{2}-q^{2}\right|>h s^{4 / 9} / 3$, a bit of algebra shows that given $A$ from Lemma 5.5, if $h$ is large enough, then

$$
\begin{aligned}
\left(p^{2}-\right. & \left.q^{2}\right)^{2} /\left(18 s^{2 / 3}\right)-A|p-q| \\
= & \left(p^{2}-q^{2}\right)^{2} /\left(36 s^{2 / 3}\right)+\left(p^{2}-q^{2}\right)^{2} /\left(36 s^{2 / 3}\right) \\
& -A|p-q| \geq h^{2} s^{2 / 9} / 324+|p-q|\left(\left|p^{2}-q^{2}\right|^{3 / 2} /\left(36 s^{2 / 3}\right)-A\right) \\
& \geq h^{2} s^{2 / 9} / 324 .
\end{aligned}
$$

The last inequality follows since $\left|p^{2}-q^{2}\right|^{3 / 2} /\left(36 s^{2 / 3}\right) \geq(h / 3)^{3 / 2} / 36$, and this quantity can be assumed to be larger than $A$. Since $\left(s^{\prime}-s\right) t^{-2 / 3}=s^{2 / 3} t^{-2 / 3} \leq 1$ we get from Lemma 5.5 the following upper bound:

$$
\begin{aligned}
\mathbf{P}_{x}\left(\sup _{s-s^{2 / 3} \leq \tau \leq s}\left|Y_{\tau}^{1}-Y_{s}^{1}\right|\right. & \left.>h s^{4 / 9}, Y_{s-s^{2 / 3}} \in B_{p}, Y_{s} \in B_{q} \mid T>t, X_{t}=x\right) \\
& \leq A a^{2} s^{4 / 3} e^{-h^{2} s^{2 / 9} / 324} .
\end{aligned}
$$

In the other case, that is, if $\left|p^{2}-q^{2}\right| \leq h s^{4 / 9} / 3$, we estimate

$$
\mathbf{P}_{x}\left(\sup _{\tau \in\left[s-s^{2 / 3}, s\right]}\left|Y_{\tau}^{1}-Y_{s}^{1}\right|>h s^{4 / 9}, Y_{s-s^{2 / 3}} \in B_{p}, Y_{s} \in B_{q}, T>t, Y_{t}=x\right)
$$

using the Markov property. For that purpose consider $y \in B_{p}, z \in B_{q}$. Assume $p \leq q$. Then, since $q \leq \sqrt{a} s^{1 / 3}$, we get

$$
\begin{aligned}
\left|y^{1}-z^{1}\right| & \leq(q+1)^{2}-p^{2}=q^{2}-p^{2}+2 q+1 \\
& \leq h s^{4 / 9} / 3+2 \sqrt{a} s^{1 / 3}+1 \leq h s^{4 / 9} / 2
\end{aligned}
$$

if $h$ is large enough. The case $q \leq p$ is similar. 
We use a Gaussian bound for

$$
\begin{aligned}
& \mathbf{P}_{y}\left(\sup _{\tau \leq s^{2 / 3}}\left|Y_{\tau}^{1}-z^{1}\right|>h s^{4 / 9}, Y_{s^{2 / 3}}=z, T>s^{2 / 3}\right) \\
\leq & \mathbf{P}_{y}\left(\sup _{\tau \leq s^{2 / 3}}\left|Y_{\tau}^{1}-y^{1}\right|>h s^{4 / 9} / 2, Y_{s^{2 / 3}}=z, T>s^{2 / 3}\right), \\
\leq & \mathbf{P}_{y^{1}}\left(\sup _{\tau \leq s^{2 / 3}}\left|Y_{\tau}^{1}-y^{1}\right|>h s^{4 / 9} / 2\right) \leq e^{-\delta h^{2} s^{2 / 9}},
\end{aligned}
$$

where $\delta>0$ is independent of $h, s$ and $y^{1}$.

The last inequality follows using the relation between the Bessel process with index 2 and the two-dimensional Brownian motion $W$. Namely

$$
\begin{gathered}
\mathbf{P}_{y^{1}}\left(\sup _{\tau \leq s^{2 / 3}}\left|Y_{\tau}^{1}-y^{1}\right|>\right. \\
\left.h s^{4 / 9} / 2\right) \leq \mathbf{P}\left(\sup _{\tau \leq s^{2 / 3}}\left|W_{\tau}-W_{0}\right|>h s^{4 / 9} / 2|| W_{0} \mid=y^{1}\right) \\
=\mathbf{P}_{0}\left(\sup _{\tau \leq s^{2 / 3}}\left|W_{\tau}\right|>h s^{4 / 9} / 2\right) .
\end{gathered}
$$

The estimate now follows from well-known properties of the maximum of the twodimensional Brownian motion.

Therefore, using the Markov property twice and the estimate (8) we obtain the following upper bound for (10):

$$
\mathcal{O}(1) e^{-\delta h^{2} s^{2 / 9}}\left|C_{q}\right| \sup _{z \in C_{q}} r_{t-s}(z, x) \int_{C_{p}} r_{s-s^{2 / 3}}(x, y) d y
$$

Now we use the symmetry relation (7) twice, Lemma 5.2 and the fact that $q^{2} \leq$ $(t-s)$ to obtain (for $p>1$ and $q>1$ ) the bound

$$
\mathcal{O}(1) p^{2} e^{A|p-q|} e^{-\delta h^{2} s^{2 / 9}} r_{t-s^{2 / 3}+\left|\tau_{p}-\tau_{q}\right|+1_{q=p} p^{2}}(x, x) .
$$

From the monotonicity of $r_{t}$ on the diagonal and Lemma [5.4, the last quantity is bounded by

$$
\mathcal{O}(1) s^{2 / 3} e^{A|p-q|} e^{-\delta h^{2} s^{2 / 9}} r_{t}(x, x)
$$

We notice that $|p-q| \leq\left|p^{2}-q^{2}\right|^{1 / 2} \leq(h / 3)^{1 / 2} s^{2 / 9}$, and therefore for large $h$ we finally get the bound for (10)

$$
\mathcal{O}(1) s^{2 / 3} e^{-\delta^{\prime} h^{2} s^{2 / 9}} r_{t}(x, x)
$$

The cases $p=1$ and/or $q=1$ first use estimate (9). The result follows by summing over $p$ and $q$ which produces an extra factor $s^{2 / 3}$.

Remark. We will use this result only for $h=s^{1 / 9}$.

Lemma 5.7. There exists $1>d>0$ such that for any $t>2$ and for any $1 \leq s \leq$ $t / 2$ we have

$$
\mathbf{P}_{x}\left(Y_{s}^{1}<s^{5 / 9} \mid T>t, X_{t}=x\right) \leq d^{-1} e^{-d s^{1 / 9}}
$$


Remark. For the general $\gamma \in(0,1)$ one gets for any $1 / 2<\beta<1 /(1+\gamma)$ the upper bound

$$
\mathbf{P}_{x}\left(Y_{s}^{1}<s^{\beta} \mid T>t, X_{t}=x\right) \leq \mathcal{O}(1) e^{-\mathcal{O}(1) s^{2 \alpha}\left(\frac{1}{1+\alpha}-\beta\right)} .
$$

Proof. By choosing the constant $d$ small, it is enough to prove the result for $t>4$ and $s>2$. Using Lemma 5.6, it is enough to give a bound on

$$
\mathbf{P}_{x}\left(\sup _{s-s^{2 / 3}<\tau<s} Y_{\tau}^{1}<2 s^{5 / 9} \mid T>t, X_{t}=x\right) .
$$

We again use the Markov property and observe that if $Y_{\tau}^{1} \leq 2 s^{5 / 9}$, we have $\left|Y_{\tau}^{2}\right| \leq$ $2 \sqrt{s^{5 / 9}-1}$, namely the process is inside the strip of width $2 \sqrt{s^{5 / 9}-1}$. Using that $Y^{2}$ is a Brownian motion and introducing the boxes in which the process is at times $s-s^{2 / 3}$ and $s$, we have for a universal constant $0<c<1$

$$
\begin{aligned}
& \mathbf{P}_{x}\left(\sup _{s-s^{2 / 3}<\tau<s} Y_{s}^{1}<2 s^{5 / 9}, T>t, Y_{t}=x\right) \\
\leq & \mathcal{O}(1) e^{-c s^{1 / 9}} \sum_{p^{2}, q^{2}=1}^{2 s^{5 / 9}} \int_{y \in B_{p}} r_{s-s^{2 / 3}}(x, y) d y \sup _{z \in B_{q}} r_{t-s}(z, x) \\
\leq & \mathcal{O}(1) e^{-c s^{1 / 9}} \sum_{p^{2}, q^{2}=2}^{2 s^{5 / 9}}(p+1)^{2} \sup _{y \in C_{p}} r_{s-s^{2 / 3}}(x, y) \sup _{z \in C_{q}} r_{t-s}(z, x),
\end{aligned}
$$

where we have used estimates (8) and (9). In order to control this quantity Lemma 5.2 is insufficient, and we will use symmetry arguments for the process with kernel $p_{t}$.

For $q>p \geq 0$, we define a sub-domain $D_{p, q}$ of $D$ by

$$
D_{p, q}=D \cap\left\{\left(p^{2}-q^{2}\right) / 2 \leq x^{1} \leq\left((p+q)^{2}+q^{2}\right) / 2\right\} \cap\left\{\left|x^{2}\right| \leq \sqrt{q^{4}-p^{4}} / 2\right\} .
$$

Let $S_{x^{i}=\sigma}$ be the symmetry with respect to the plane $x^{i}=\sigma$. Using the maximum principle, it follows easily that for any $y \in D_{p, q}$, we have for any $\tau>1$

$$
\begin{aligned}
p_{\tau}(x, y) \leq & p_{\tau}^{D_{p, q}}(x, y)+p_{\tau}\left(x, S_{x^{1}=\left(p^{2}-q^{2}\right) / 2}(y)\right)+p_{\tau}\left(x, S_{x^{1}}=\left((p+1)^{2}+q^{2}\right) / 2\right. \\
& +(y)) \\
& +p_{\tau}\left(x, S_{x^{2}=\sqrt{q^{4}-p^{4}} / 2}(y)\right)+p_{\tau}\left(x, S_{x^{2}=-\sqrt{q^{4}-p^{4}} / 2}(y)\right)
\end{aligned}
$$

where $p_{\tau}^{D_{p, q}}(x, y)$ denotes the heat kernel in $D_{p, q}$ with Dirichlet boundary conditions extended by zero outside.

Using $D_{p, q} \subset\left\{\left|x^{3}\right| \leq 2 q+1\right\}$ we get by comparison of the heat kernels and for any $t>1$

$$
p_{\tau}^{D_{p, q}}(x, y) \leq c^{-1} e^{-c \tau /(q+1)^{2}}
$$


where $0<c<1$ is a constant independent of $x$ and $y$. We conclude that for any $y \in C_{p}$ we have

$$
\begin{gathered}
r_{\tau}(x, y) \\
\leq c^{-1} q^{2} e^{-c \tau /(q+1)^{2}}+y^{1} \int_{0}^{2 \pi} d \theta p_{\tau}\left(\left(x^{1} \cos \theta, x^{2} \sin \theta, x^{3}\right), S_{x^{1}=\left(p^{2}-q^{2}\right) / 2}\left(\left(y^{1}, 0, y^{2}\right)\right)\right) \\
+y^{1} \int_{0}^{2 \pi} d \theta p_{\tau}\left(\left(x^{1} \cos \theta, x^{2} \sin \theta, x^{3}\right), S_{x^{1}=\left((p+1)^{2}+q^{2}\right) / 2}\left(\left(y^{1}, 0, y^{2}\right)\right)\right) \\
+y^{1} \int_{0}^{2 \pi} d \theta p_{\tau}\left(\left(x^{1} \cos \theta, x^{2} \sin \theta, x^{3}\right), S_{x^{2}=\sqrt{q^{4}-p^{4}} / 2}\left(\left(y^{1}, 0, y^{2}\right)\right)\right) \\
+y^{1} \int_{0}^{2 \pi} d \theta p_{\tau}\left(\left(x^{1} \cos \theta, x^{2} \sin \theta, x^{3}\right), S_{x^{2}=-\sqrt{q^{4}-p^{4}} / 2}\left(\left(y^{1}, 0, y^{2}\right)\right)\right)
\end{gathered}
$$

By a simple geometric argument, it follows from the choice of the symmetries (and hence of $D_{p, q}$ ) that if $y \in C_{p}$, then all the four points

$$
S_{x^{1}=\left((p+1)^{2}+q^{2}\right) / 2}\left(\left(y^{1}, 0, y^{2}\right)\right), S_{x^{1}=\left(q^{2}-p^{2}\right) / 2}\left(\left(y^{1}, 0, y^{2}\right)\right),
$$

and

$$
S_{x^{2}= \pm \sqrt{q^{4}-p^{4}} / 2}\left(\left(y^{1}, 0, y^{2}\right)\right)
$$

have in cylindrical coordinates a polar radius between $q^{2}$ and $(q+1)^{2}$, i.e. each of them is of the form $\left(\rho \cos \phi, \rho \sin \phi, y^{2}\right)$ for some $q^{2} \leq \rho \leq(q+1)^{2}$ and $0 \leq \phi<2 \pi$. Therefore by the rotation invariance of the kernel $p_{\tau}$ we conclude that for any $\tau>1$ and any $q>p$

$$
\sup _{y \in C_{p}} r_{\tau}(x, y) \leq 4 \sup _{y \in C_{q}} r_{\tau}(x, y)+c^{-1} q^{2} e^{-c \tau /(q+1)^{2}} .
$$

Therefore, if $q>p$ we have

$$
\begin{gathered}
\sup _{y \in C_{p}} r_{s-s^{2 / 3}}(x, y) \sup _{y \in C_{q}} r_{t-s}(y, x) \\
\leq 4 \sup _{y \in C_{q}} r_{s-s^{2 / 3}}(x, y) \sup _{y \in C_{q}} r_{t-s}(y, x)+c^{-1} q^{2} e^{-c s / 2(q+1)^{2}} \sup _{y \in C_{q}} r_{t-s}(y, x) .
\end{gathered}
$$

Using Harnack's inequality and averaging over a disk of radius one, the first term is bounded by

$$
\mathcal{O}(1) r_{t-s^{2 / 3}+q^{2}}(x, x),
$$

and we can use Lemma 5.4 to show that this is smaller than $\mathcal{O}(1) r_{t}(x, x)$. For the remainder term, we use Harnack's inequality $q+\mathcal{O}(1)$ times to get

$$
\sup _{y \in C_{q}} r_{t-s}(y, x) \leq \mathcal{O}(1) C^{q} r_{t-s+q^{3}}(x, x) \leq \mathcal{O}(1) C^{q} r_{t}(x, x) e^{E s t^{-2 / 3}},
$$

where the last inequality follows using Lemma 5.4. Therefore, if $q>p$ we get

$$
\begin{gathered}
\sup _{y \in C_{p}} r_{s-s^{2 / 3}}(x, y) \sup _{y \in C_{q}} r_{t-s}(y, x) \\
\leq \mathcal{O}(1) r_{t}(x, x)+C^{q} c^{-1} q^{2} e^{-c s / 2(q+1)^{2}} r_{t}(x, x) e^{E s t^{-2 / 3}} \leq \mathcal{O}(1) r_{t}(x, x)
\end{gathered}
$$

since $q^{2}<\mathcal{O}(1) s^{5 / 9}$. 
If $q<p$ we have

$$
\begin{gathered}
\sup _{y \in C_{p}} r_{s-s^{2 / 3}}(x, y) \sup _{y \in C_{q}} r_{t-s}(y, x) \\
\leq 4 \sup _{y \in C_{p}} r_{s-s^{2 / 3}}(x, y) \sup _{y \in C_{p}} r_{t-s}(y, x)+c^{-1} p^{2} e^{-c(t-s) /(p+1)^{2}} \sup _{y \in C_{p}} r_{s-s^{2 / 3}}(x, y) .
\end{gathered}
$$

Using Harnack's inequality, and averaging over a disk of radius one, the first term is bounded by

$$
\mathcal{O}(1) r_{t-s^{2 / 3}+p^{2}}(x, x),
$$

and we can use Lemma 5.4 to show that this is smaller than $\mathcal{O}(1) r_{t}(x, x)$. For the remainder term, we have since $p^{2}<2 s^{5 / 9}$

$$
p^{2} e^{-c(t-s) /(p+1)^{2}} \leq s^{2 / 3} e^{-c t /\left(4 s^{5 / 9}\right)} \leq t^{2 / 3} e^{-c t^{4 / 9} / 4} \leq \mathcal{O}(1) r_{t}(x, x),
$$

where the last inequality follows from the second part of Lemma 5.2 Therefore in this case we also get

$$
\sup _{y \in C_{p}} r_{s-s^{2 / 3}}(x, y) \sup _{y \in C_{q}} r_{t-s}(x, y) \leq \mathcal{O}(1) r_{t}(x, x) .
$$

The proof is completed by using (11).

The proof of Lemma 2.1 will be completed with the following result.

Lemma 5.8. We have for any $M>0$

$$
\limsup _{t \rightarrow+\infty} \mathbf{E}_{x}\left(\int_{M}^{t-M} \frac{d s}{\left(Y_{s}^{1}\right)^{2}} \mid T>t, Y_{t}=x\right)<\infty
$$

and

$$
\lim _{M \rightarrow+\infty} \limsup _{t \rightarrow+\infty} \mathbf{E}_{x}\left(\int_{M}^{t-M} \frac{d s}{\left(Y_{s}^{1}\right)^{2}} \mid T>t, Y_{t}=x\right)=0 .
$$

Proof. Using (7), it follows easily that for $u>0$ and $s>0$ we have

$$
r_{s}(x, y) r_{u}(y, x)=r_{u}(x, y) r_{s}(y, x) .
$$

Therefore, it is enough to consider the integral from $M$ to $t / 2$ (and $t>2 M$ ).

We split the expectation into two parts. From Lemma 5.7 we have

$$
\begin{gathered}
\mathbf{E}_{x}\left(\frac{1}{\left(Y_{s}^{1}\right)^{2}}, Y_{s}^{1}<s^{5 / 9} \mid T>t, Y_{t}=x\right) \\
\leq \mathbf{P}_{x}\left(Y_{s}^{1} \leq s^{5 / 9} \mid T>t, X_{t}=x\right) \leq d^{-1} e^{-d s^{1 / 9}} .
\end{gathered}
$$

On the other hand

$$
\mathbf{E}_{x}\left(\frac{1}{\left(Y_{s}^{1}\right)^{2}}, Y_{s}^{1} \geq s^{5 / 9} \mid T>t, Y_{t}=x\right) \leq \mathcal{O}(1) s^{-10 / 9}
$$

Finally, we obtain

$$
\mathbf{E}_{x}\left(\frac{1}{\left(Y_{s}^{1}\right)^{2}} \mid T>t, Y_{t}=x\right) \leq \mathcal{O}(1) s^{-10 / 9},
$$

and the lemma follows. 
5.2. Proof of Theorem 1.5. We start by showing the first equality in the theorem. We point out that from the asymptotic ratio behavior for $q_{t}^{0}$ (see (4)) we obtain the corresponding behavior for $r_{t}$,

$$
\lim _{t \rightarrow \infty} \frac{r_{t}(x, y)}{r_{t}\left(x_{0}, y_{0}\right)}=\frac{u_{0}(x) u_{0}(y) y^{1}}{u_{0}\left(x_{0}\right) u_{0}\left(y_{0}\right) y_{0}^{1}} .
$$

Consider $x$, with $x^{2}=0$, and define

$$
\beta(t)=\sup _{y} r_{t}(x, y) .
$$

By reflection with respect to the second coordinate of $y$ it follows that the maximum is located at a point (or several) of the form $y_{t}=\left(y_{t}^{1}, 0\right)$. On the other hand, from (12), (9) and the fact that $u_{0}$ vanishes in the boundary of $D$, it follows that $d\left(y_{t}, \partial D\right)$ is bounded away from 0 . The Gaussian bound given in Lemma 5.1 and the lower bound on $r_{t}(x, x)$ from Lemma 5.2 imply that $y_{t}^{1} \leq C t^{2 / 3}$ for some constant $C>0$. For any $\tilde{C}>C$, we have again from the Gaussian bound of Lemma 5.1

$$
\begin{aligned}
\mathbf{P}_{x}(T>t) & =\int_{\substack{y^{1} \leq \tilde{C} t^{2 / 3}\\
}} r_{t}(x, y) d y+\int_{\substack{y^{1} \geq \tilde{C} t^{2 / 3}\\
}} r_{t}(x, y) d y \\
& \leq \tilde{C}^{3 / 2} t \beta(t)+\mathcal{O}(1) e^{-\tilde{C}^{2} t^{1 / 3} / 4} .
\end{aligned}
$$

Therefore, from the lower bound on $\beta(t)\left(\geq r_{t}(x, x)\right)$ coming from Lemma 5.2, we can choose $\tilde{C}$ large enough such that the first term of this last inequality dominates the second one, and we obtain

$$
\mathbf{P}_{x}(T>t) \leq \mathcal{O}(1) t \beta(t) .
$$

On the other hand, using Harnack's inequality in a ball $B\left(y_{t-1}, 2 R\right)$ around $y_{t-1}$ (with $R$ small enough such that the ball is inside $D$ ), we have

$$
\beta(t-1) \leq \mathcal{O}(1) \int_{B\left(y_{t-1}, R\right)} r_{t}(x, y) d y \leq \mathcal{O}(1) \mathbf{P}_{x}(T>t)
$$

Using the lower bound on $r_{t}(x, y)$ from Lemma 5.2 and the Gaussian bound we get

$$
\begin{gathered}
r_{t}(x, x)=\int r_{t / 2}(x, z) r_{t / 2}(z, x) d z \\
\leq \int_{z^{1}<\mathcal{O}(1) t^{2 / 3}} r_{t / 2}(x, z) r_{t / 2}(z, x) d z+o(1) r_{t}(x, x) .
\end{gathered}
$$

Now, from the symmetry property (17) verified by $r_{t}$ we get

$$
r_{t}(x, x) \leq \mathcal{O}(1) t \beta(t / 2)^{2} .
$$

Again Harnack's inequality and the symmetry properties of $r$ give

$$
\begin{gathered}
\beta(t / 2-1)^{2} \leq \mathcal{O}(1) \int_{B\left(y_{t / 2-1}, R\right)} r_{t / 2}(x, z)^{2} d z \\
\leq \mathcal{O}(1) t^{2 / 3} \int_{B\left(y_{t / 2-1}, R\right)} r_{t / 2}(x, z) r_{t / 2}(z, x) d z=\mathcal{O}(1) t^{2 / 3} r_{t}(x, x),
\end{gathered}
$$

and we have proved

$$
\mathcal{O}(1) t^{-2 / 3} \beta(t / 2-1)^{2} \leq r_{t}(x, x) \leq \mathcal{O}(1) t \beta(t / 2)^{2} .
$$


Note also that by the Gaussian bound for any $\tau>2$

$$
r_{\tau}(x, y)=\int r_{\tau-1}(x, z) r_{1}(z, y) d z \leq \mathcal{O}(1) \beta(\tau-1)
$$

and hence $\beta(t / 2) \leq \mathcal{O}(1) \beta(t / 2-1)$, which implies

$$
\mathcal{O}(1) t^{-2 / 3} \beta(t / 2-1)^{2} \leq r_{t}(x, x) \leq \mathcal{O}(1) t \beta(t / 2-1)^{2} .
$$

It also follows immediately from (13) and (14) that

$$
\mathcal{O}(1) \beta(t-1) \leq \mathbf{P}_{x}(T>t) \leq \mathcal{O}(1) t \beta(t-1) .
$$

Combining all the bounds we get

$$
\lim _{t \rightarrow \infty} t^{-1 / 3} \log \left(\frac{r_{2 t}(x, x)^{1 / 2}}{\mathbf{P}_{x}(T>t)}\right)=0 .
$$

For a fixed $x=\left(x^{1}, 0, x^{3}\right) \in D$, we denote by $\mathcal{C}(x)$ the circle obtained by rotating $x$ around the vertical axis. We can cover this circle by a finite number of balls whose radius is smaller than one fourth of their distance to the boundary of $D$. Applying Harnack's inequality in balls twice as big, we obtain that there is a constant $C>0$ (which may depend on $x$ ) such that for any $t>2$ and any $y \in \mathcal{C}$ we have

$$
C^{-1} p_{t-1}(x, y) \leq p_{t}(x, x) \leq C p_{t+1}(x, y) .
$$

Integrating along $\mathcal{C}$ we get

$$
C^{-1} r_{t-1}(x, x) \leq 2 \pi x^{1} p_{t}(x, x) \leq C r_{t+1}(x, x) .
$$

Since (see [4]) for any fixed $\tau$

$$
\lim _{t \rightarrow \infty} \frac{p_{t}(x, x)}{p_{t+\tau}(x, x)}=1,
$$

we get

$$
\lim _{t \rightarrow \infty} t^{-1 / 3} \log \left(\frac{p_{2 t}(x, x)^{1 / 2}}{\mathbf{P}_{x}(T>t)}\right)=0,
$$

and the first equality in Theorem 1.5 is proved.

Now we establish the second equality of the theorem. Let $\bar{q}_{t}$ be the heat kernel of a two-dimensional Brownian motion in $\mathcal{P}$. The Feynmann-Kac formula gives

$$
\bar{q}_{t}(x, y)=r_{t}(x, y) \sqrt{\frac{x^{1}}{y^{1}}} \mathbf{E}_{x}\left(e^{-\frac{3}{8} \int_{0}^{t} \frac{1}{Y_{s}^{12}} d s} \mid Y_{t}=y, T>t\right),
$$

and the Jensen inequality implies

$$
1 \geq \frac{\bar{q}_{t}(x, x)}{r_{t}(x, x)} \geq \exp \left(-\frac{3}{8} \mathbf{E}_{x}\left(\int_{0}^{t} \frac{1}{Y_{s}^{1^{2}}} d s \mid Y_{t}=x, T>t\right)\right) .
$$

Let $\bar{u}$ be a nontrivial $\Delta$-harmonic function in the parabola with Dirichlet boundary conditions. The Martin boundary at infinity for the Laplacian in the parabola has dimension one. This follows from the fact that the conformal mapping of the interior of the parabola to the upper half plane is a bijection at the boundary including the points at infinity (see for example [14] for the explicit formula). Therefore the Martin boundary at infinity of the parabola is the same as the Martin boundary at infinity of the upper half plane which has dimension one (see [6]). 
From the asymptotic ratio behavior for $r_{t}$, the bounds in (16) and the results in [4], we deduce that for any sequence $\left(t_{n}\right)$ there exists a subsequence $\left(t_{n^{\prime}}\right)$ and a positive constant $A>0$ (which may depend on the subsequence) such that

$$
\lim _{t_{n^{\prime}} \rightarrow \infty} \frac{\bar{q}_{t_{n^{\prime}}}(x, y)}{r_{t_{n^{\prime}}}(x, y)}=A \frac{\bar{u}(x) \bar{u}(y)}{u_{0}(x) u_{0}(y) y^{1}} \quad \text { for all } x, y \in \mathcal{P} .
$$

Theorem 1.4 and (16) show that the following limit exists:

$$
A \lim _{\rho \rightarrow \infty} \frac{\bar{u}^{2}(\rho, 0)}{u_{0}^{2}(\rho, 0) \rho}=1
$$

Since the Martin boundary at infinity for the Laplacian in the parabola has dimension one, we can choose $\bar{u}$ in order that

$$
\lim _{\rho \rightarrow \infty} \frac{\bar{u}(\rho, 0)}{u_{0}(\rho, 0) \sqrt{\rho}}=1 .
$$

With this normalization we obtain

$$
\lim _{t_{n^{\prime}} \rightarrow \infty} \frac{\bar{q}_{t_{n^{\prime}}}(x, y)}{r_{t_{n^{\prime}}}(x, y)}=\frac{\bar{u}(x) \bar{u}(y)}{u_{0}(x) u_{0}(y) y^{1}} .
$$

Since the limit does not depend on the subsequence we conclude that

$$
\lim _{t \rightarrow \infty} \frac{\bar{q}_{t}(x, y)}{r_{t}(x, y)}=\frac{\bar{u}(x) \bar{u}(y)}{u_{0}(x) u_{0}(y) y^{1}} .
$$

On the other hand we also deduce that

$$
\lim _{t \rightarrow \infty} \frac{\log \left(\bar{q}_{t}(x, y)\right)}{\log \left(r_{t}(x, y)\right)}=1
$$

and from [17] we deduce that

$$
\lim _{t \rightarrow \infty} t^{-1 / 3} \log \left(r_{t}(x, y)\right)=-2^{2 / 3} C_{1 / 2}
$$

where $C_{1 / 2}$ is the constant appearing in formula (12) of [15]. From Harnack's inequality (see (15)) the same asymptotic follows for $p_{t}$.

This ends the proof of Theorem 1.5 .

\section{ACKNOWLEDGMENTS}

The authors thank the Nucleus Millennium Information and Randomness P01005 for support. The first author would like to thank the CMM for its hospitality. We also thank the referee for her/his valuable comments that improved the exposition of this paper.

\section{REFERENCES}

[1] Ancona, A. Théorie du potentiel sur les graphes et les variétés. Ecole de Probabilités de SaintFlour XVIII-1988, 1-112, Lecture Notes in Math., 1427, Springer, Berlin, 1990. MR.1100282 (92g:31012)

[2] Aikawa, H.; Essén, M. Potential theory-selected topics. Lecture Notes in Mathematics, 1633, Springer-Verlag, Berlin, 1996. MR,1439503 (98f:31005)

[3] Bañuelos, R.; DeBlassie, R.; Smits, R. The first exit time of planar Brownian motion from the interior of a parabola. Ann. Probab. 29 (2001), no. 2, 882-901. MR.1849181 (2002h:60165)

[4] Collet, P.; Martínez, S.; San Martín, J. Ratio limit theorems for a Brownian motion killed at the boundary of a Benedicks domain. Ann. Probab. 27 (1999), no. 3, 1160-1182. MR1733144 (2001d:60089) 
[5] Collet, P.; Martínez, S.; San Martín, J. Asymptotic of the heat kernel in general Benedicks domains. Probab. Theory Related Fields 125 (2003), no. 3, 350-364. MR.1964457(2004d:60209)

[6] Deitmar, A. A first course in harmonic analysis. Universitext. Springer-Verlag, New York, 2002. MR1885996 (2003b:42001)

[7] Doob, J. L. Classical potential theory and its probabilistic counterpart. Grundlehren der Mathematischen Wissenschaften, 262, Springer-Verlag, New York, 1984. MR0731258 (85k:31001)

[8] Ioffe, D.; Pinsky, R. Positive harmonic functions vanishing on the boundary for the Laplacian in unbounded horn-shaped domains. Trans. Amer. Math. Soc. 342 (1994), no. 2, 773-791. MR.1211410 (94h:60114)

[9] Lifshits, M.; Shi, Z. The first exit time of Brownian motion from a parabolic domain. Bernoulli 8 (2002), no. 6, 745-765. MR.1963660 (2004d:60213)

[10] Moser, J. A Harnack inequality for parabolic differential equations. Comm. Pure Appl. Math. 17 (1964), 101-134. MR0159139 (28:2357)

[11] Maz'ya, V. G. On the relationship between Martin and Euclidean topologies. Soviet Math. Dokl. 18 (1977), 283-286.

[12] Murata, M. Semismall perturbations in the Martin boundary theory for elliptic equations. Israel Journal of Mathematics 102 (1997), 29-60. MR.1489100 (98k:35036)

[13] Murata, M. Martin boundaries of elliptic skew products, semismall perturbations, and fundamental solutions of parabolic equations. J. Funct. Anal. 194 (2002), no. 1, 53-141. MR1929139 (2003i:35052)

[14] Nehari, Z. Conformal mapping. Reprinting of the 1952 edition. Dover Publications, Inc., New York, 1975. MR0377031 (51:13206)

[15] Ruelle, D. Probability estimates for continuous spin systems. Comm. Math. Phys. 50 (1976), no. 3, 189-194. MR0424129 (54:12097)

[16] Revuz, D.; Yor, M. Continuous martingales and Brownian motion. Third edition. Grundlehren der Mathematischen Wissenschaften, 293, Springer-Verlag, Berlin, 1994.

[17] van den Berg, M. Subexponential behaviour of the Dirichlet heat kernel. J. Funct. Anal. 198 (2003), no. 1, 28-42. MR1962352 (2003m:60211)

Centre de Physique Théorique, CNRS-UMR 7644 Ecole Polytechnique, 91128 Palaiseau Cedex, France

E-mail address: Pierre.Collet@cpht.polytechnique.fr

CMM-Dim, UMi 2807-CNRS, Universidad de Chile, Casilla 170-3 Correo 3, Santiago, CHILE

E-mail address: smartine@dim.uchile.cl

CMM-Dim, UMi 2807-CNRS, Universidad de Chile, Casilla 170-3 Correo 3, Santiago, CHILE

E-mail address: jsanmart@dim.uchile.cl 